\title{
Type Ia Supernovae: An Examination of Potential Progenitors and the Redshift Distribution
}

\author{
L. Yungelson円 and M. Livio \\ Space Telescope Science Institute \\ 3700 San Martin Drive, Baltimore, MD 21218
}

Received —

\footnotetext{
${ }^{1}$ Permanent address: Institute of Astronomy of the Russian Academy of Sciences, 48 Pyatnitskaya Str., 109017 Moscow, Russia
} 


\begin{abstract}
We examine the possibility that supernovae type Ia (SN Ia) are produced by white dwarfs accreting from Roche-lobe filling evolved companions, under the assumption that a strong optically thick stellar wind from accretor is able to stabilize the mass transfer. We show that if a mass transfer phase on a thermal timescale precedes a nuclear burning driven phase, then such systems (of which the supersoft X-ray sources are a subgroup) can account for about $10 \%$ of the inferred SN Ia rate.

In addition, we examine the cosmic history of the supernova rate, and we show that the ratio of the rate of SN Ia to the rate of supernovae produced by massive stars (supernovae of types II, Ib, Ic) should increase from about $z=1$ towards lower redshifts.
\end{abstract}

Subject headings: binaries: close-binaries: symbiotic-stars: mass-loss-stars: novae-supernovae: general-X-rays: stars-galaxies: evolution 


\section{INTRODUCTION}

Type Ia supernovae (SN Ia) are believed to result from the thermonuclear disruptions of accreting C-O white dwarfs (WDs) in binary systems. However, their immediate progenitors have not yet been identified. Two main scenarios are currently discussed most frequently. In one, the exploding white dwarf reaches the Chandrasekhar mass, $M_{C h} \approx 1.4 M_{\odot}$, and carbon ignition occurs in the WD's center. In the second, the accreted layer of helium on top of a C-O WD ignites off-center, at sub-Chandrasekhar masses (edge-lit detonations, henceforth, ELD). For the most recent reviews of the different aspects of the evolutionary scenarios which may result in potentially explosive configurations see e.g. Branch et al. (1995), Wheeler (1996), Livio (1996a), Ruiz-Lapuente, Canal, \& Burkert (1997), Yungelson \& Tutukov (1997).

The double degenerate scenario (Webbink 1984; Iben \& Tutukov 1984), in which the exploding object is of a Chandrasekhar mass or higher, is one of the two evolutionary channels for which estimates based on population synthesis studies (see Yungelson \& Tutukov 1997 and references therein) give occurrence rates of SN Ia of the same order as those inferred for the Milky Way Galaxy: $\sim 10^{-3} \mathrm{yr}^{-1}$ (Cappellaro et al. 1997). This channel is able to account for the occurrence of SN Ia both in young and old stellar populations. In the second channel, the accretion of helium from a Roche-lobe filling nondegenerate helium star may result in an ELD (Livne \& Glasner 1990; Woosley \& Weaver 1994; Livne \& Arnett 1995) in sub-Chandrasekhar WDs at comparable rates. However, this channel may operate only in relatively young populations (age $\lesssim 10^{9} \mathrm{yr}$ ), and it is not clear if it can account for the high-velocity intermediate-mass elements that are seen in SN Ia spectra (see e.g. Branch et al. 1995 for a discussion). The double degenerate scenario on the other hand is plagued by two basic weaknesses. First, no close binary white dwarf system with a total mass higher than $M_{C h}$ has been found yet. While Iben, Tutukov, \& Yungelson (1997) have shown that the fraction of close binary carbon-oxygen white dwarfs with $M_{1}+M_{2} \gtrsim M_{C h}$ among the potentially observable pairs hardly exceeds $\sim 1 / 200$, observations are beginning to place meaningful constraints on this scenario (e.g. Saffer \& Livio 1997).

Second, existing calculations of the merger process (e.g. Benz et al. 1990; Mochkovich \& Livio 1990; Mochkovich, Guerrero, \& Segretain 1997; Segretain, Chabrier, \& Mochkovich 1997) tend to indicate that the outcome of the merger may be an accretion induced collapse rather than an explosion, or the formation of a single white dwarf. The observational counterparts of the systems in which the accretion of helium from nondegenerate stars occurs are also not known, although hot blue subdwarfs are prime candidates.

The circumstances listed in the preceding paragraphs stimulated the search for other channels which could lead to a sufficiently high occurrence rate of SN Ia. In particular, the interest in the systems in which white dwarfs accrete hydrogen at high rates, as potential progenitors, has increased substantially (e.g. Di Stefano 1996; Livio 1996a; Yungelson et al. 1996; Di Stefano et al. 1997; Li \& van den Heuvel 1997). These systems may show up 
as supersoft X-ray sources (see Kahabka \& van den Heuvel 1997 for a review). Recently, Hachisu, Kato, \& Nomoto (1996, henceforth HKN) suggested what they termed as "a new model for the progenitor systems of type Ia supernovae". This model envisions a massive white dwarf accreting from a Roche-lobe filling, well evolved $\left(M_{\text {He core }} \gtrsim 0.35 M_{\odot}\right)$, low-mass red giant. In fact, such configurations were suggested as potential SN Ia progenitors as early as 1973 (Whelan \& Iben 1973) and were considered by Iben \& Tutukov (1984) and Yungelson et al. (1996, henceforth, YLTTF). The latter authors have shown that in this model the rate of explosions of Chandrasekhar mass WDs is more than an order of magnitude below the desired one. However, HKN argued that with certain modifications to the input physics, this model can produce a Galactic rate of SN Ia of $\sim 0.002 \mathrm{yr}^{-1}$ (although this number was obtained as a rather crude estimate, without a detailed modeling of the underlying population).

In the present work we incorporate the modifications of HKN into a population synthesis code and thereby estimate the occurrence rate of SN Ia due to this mechanism. We show that even under the most favorable assumptions, this channel is not able to produce more than $\sim 10^{-4}$ events per year. We suggest however some modifications to the original HKN scenario which somewhat increase its efficiency. Our implementation of the HKN model and the suggested modifications are described in $\S 2$. The results are presented and discussed in $\S 3$. In $\S 4$ we examine the question of SN Ia from a cosmic perspective, in view of recent findings on the history of the star formation rate in the universe. Our conclusions follow.

\section{THE MODEL}

\subsection{The Hashisu-Kato-Nomoto model}

The essence of the HKN model may be described as follows. As it was noticed by Refsdal \& Weigert $(1970,1971)$, the radii, luminosities, and core growth rates of stars with degenerate helium cores depend mainly on the core mass itself. The same is true for stars with degenerate carbon-oxygen cores (Paczyński 1970, Uus 1970). This allows to follow the evolution of thermally stable subgiant stars, both underfilling and filling their tidal lobes, without detailed evolutionary computations. The stars (of mass $M_{2}$ and radius $R_{2}$ ) which continuously fill their Roche-lobes have to satisfy the following criteria:

$$
R_{2}=R_{c r} \quad \text { and } \quad \frac{d \ln R_{2}}{d \ln M_{2}}=\frac{d \ln R_{c r}}{d \ln M_{2}}
$$

where $R_{c r}$ is the radius of the Roche-lobe. If some of the matter which is transferred from the donor to the accretor is lost by the latter, taking away the specific angular momentum of the accretor, Eq. (1) gives for the mass loss rate by the donor [this Eq. is equivalent to 
Eq. (5) of HKN]:

$$
\frac{\dot{M}_{2}}{M_{2}}=-\frac{1}{2} \frac{\dot{R}}{R} \times\left[1-\alpha q-\frac{1}{2} \frac{1-\alpha}{1+q} q-\frac{1-\alpha}{1+q} q^{2}-\frac{1}{2}(1+\alpha q) f^{\prime}\right]^{-1} .
$$

Here $q=M_{2} / M_{1}$ is the mass ratio, $f^{\prime}$ is the logarithmic derivative of the relative radius of the Roche lobe, and $\alpha$ is the fraction of the transferred matter which is retained by the accretor (retention efficiency). If $\alpha$ is close to 1 , Eq. (2) is valid for $q \lesssim 0.78$. For larger values of $q$, the denominator in Eq. (2) becomes negative and the expression gives a positive value of $\dot{M}_{2} / M_{2}$. This means that under these conditions mass transfer proceeds on a thermal or dynamical timescale (e.g. Hjellming \& Webbink 1987; Soberman, Phinney, \& van den Heuvel 1997). In the conventional model of evolution, this implies that the accretion rate exceeds the maximum rate at which the WD can burn the accreted hydrogen stably, $\dot{M}_{c r} \sim 10^{-7} \mathrm{M}_{\odot} \mathrm{yr}^{-1}$. A common envelope forms, engulfing the entire system, and the subsequent evolution may lead to the formation of a double degenerate system or to a merger of the components. For binaries which satisfy the criterion $q \lesssim 0.78$, Eq. (2) was applied by e.g. Yungelson (1973), Webbink, Rappaport, \& Savonije (1983), Iben \& Tutukov (1984), Kraicheva et al. (1986), de Kool, van den Heuvel, \& Rappaport (1986), and Yungelson et al. $(1995,1996)$ to a number of problems related to the evolution of Algols, low-mass X-ray binaries, symbiotic stars, and supersoft X-ray sources.

HKN suggested that an optically thick stellar wind from the white dwarf (Kato \& Hachisu 1994) may stabilize the mass transfer for systems with $q \gtrsim 0.78$. An optically thick wind may blow off the excess of matter above $\dot{M}_{c r}$ at a rate of up to $\sim 10^{-4} \mathrm{M}_{\odot} \mathrm{yr}^{-1}$. The velocity of the wind may be as high as $\sim 10^{3} \mathrm{~km} \mathrm{~s}^{-1}$ and its specific angular momentum is that of the accretor. If an optically thick wind indeed operates, $\alpha$ may become close to 0 . In such a case, Eq. (2) becomes valid for $q \lesssim 1.15$. This could in principle increase the number of systems which undergo stable mass transfer. From an evolutionary point of view it should be noted that frictional drag inside the wind is not able to significantly affect the orbit of the companion star (e.g. Kato \& Hachisu 1994).

HKN performed a set of test integrations of (their version of) Eq. (2) for a number of combinations of donor and accretor masses. They have shown that in systems with initial donor masses of $M_{d} \approx 0.8-1.4 M_{\odot}$, (with He core masses $M_{H e 0} \geq 0.35 M_{\odot}$ ), and accretor masses $M_{a} \approx 1.0-1.2 M_{\odot}$, which obey the criterion $q=M_{d} / M_{a} \leq 1.15$, the accretors can actually grow in mass to $M_{C h}$.

\subsection{Modifications to the Hachisu-Kato-Nomoto model}

Let us introduce $\xi_{L}=\frac{d \ln R_{2}}{d \ln M_{2}}$ for the mass derivative of the Roche-lobe radius, $\xi_{a d}=\left(\frac{\partial \ln R_{2}}{\partial \ln M_{2}}\right)_{a d}$ for the adiabatic derivative of the stellar radius and $\xi_{t h}=\left(\frac{\partial \ln R_{2}}{\partial \ln M_{2}}\right)_{t h}$ for the derivative of the radius of the star in thermal equilibrium. Dynamical stability of the mass 
exchange process requires $\xi_{L}<\xi_{a d}$, and thermal stability requires $\xi_{L}<\xi_{\text {th }}$ (e.g. Ritter 1996). For fully convective stars $\xi_{a d}=-1 / 3$. However, Hjellming and Webbink (1987) have shown, by an analysis of polytropic models, that in the presence of a condensed radiative core, $\xi_{a d}$ may be significantly higher than $-1 / 3$. Giant branch stars with masses of degenerate cores $M_{H e} \gtrsim 0.25$ can be adequately approximated by polytropes with condensed radiative cores and convective envelopes. Stars with less massive cores have relatively shallow convective envelopes and for them the criteria for stability derived for stars with radiative envelopes are more appropriate. In Fig. 1 we plot the dependence of $\xi_{L}$ on $q$ for two extreme values of the retention efficiency $\alpha=0$ and 1 . As an example (appropriate for the present study), we compare $\xi_{L}$ with $\xi_{a d}$ for two values of the fractional mass of the condensed core: $m_{c} \approx 0.2$ and $m_{c} \approx 0.39$ (taken from Hjellming \& Webbink 1987). It is clear that for values of $\alpha$ close to 0 , Roche-lobe filling giants may be dynamically stable even for values of $q$ much higher than 1.15. However, if $\xi_{t h}<\xi_{L}<\xi_{a d}$ these stars are thermally unstable. For giants with radii and luminosities independent of mass, $\xi_{t h}=0$. This analysis suggests to include as candidate SN Ia progenitors also stars which lose mass on a thermal timescale. This means that we have to reject from the sample of candidate systems only those systems whose donors had $M_{H e}>0.25 M_{\odot}$ and $\xi_{L}>\xi_{a d}$ (for the appropriate values of $q$ and $m_{c}$ ).

Existing calculations of the mass exchange in low-mass binaries with donors possessing a deep convective envelope do not provide sufficient information on the time-dependence of $\dot{M}_{2}$. We therefore applied the following simplified algorithm (see also Rappaport, Di Stefano, \& Smith 1994). We estimated the mass loss rate, $\dot{M}_{t h}$, which corresponded to the thermal timescale of the donor at the instant of Roche Lobe overflow (RLOF) and assumed that the star loses mass at a constant rate $\dot{M}_{2}=\alpha_{t h} \dot{M}_{t h}$ until Eq. (2) becomes valid. Several sets of runs were performed for values in the range $0.1 \leq \alpha_{t h} \leq 2$. Systems with initial transfer rates of $\left|\dot{M}_{2}\right|>10^{-4} \mathrm{M}_{\odot} \mathrm{yr}^{-1}$ were rejected, because for them, optically thick wind solutions do not exist (Kato \& Hachisu 1994).

In addition, we calculated the occurrence rates of events in which ONe white dwarfs reached $M_{C h}$. While these events are expected to produce accretion induced collapses (AIC) rather than SN Ia (Canal \& Shatzman 1976), they are of great interest for the formation of low-mass X-ray binaries. In our population synthesis code, the ONe white dwarfs are assumed to descend from stars with MS masses between 9 and $11.4 M_{\odot}$. The width of this mass interval may be somewhat overestimated, however it is quite clear that stars with masses of 10-10.5 $M_{\odot}$ do produce ONe white dwarfs (e.g. Dominguez, Tornambè, \& Isern 1993; Ritossa, Garcia-Berro, \& Iben 1996). The minimum mass of an ONe white dwarf in our code is $1.19 M_{\odot}$. Actually, cold and massive $\left(M \gtrsim 1 M_{\odot}\right)$ CO white dwarfs may also experience AICs instead of supernova explosions (e.g. Nomoto \& Kondo 1991; Canal \& Gutierrez 1997). The mass range of CO white dwarfs able to collapse is strongly dependent on the approximations adopted for, e.g. the thermal structure of the WD and the nuclear reaction rates. However, with this in mind, we have to note that the rate of events considered below as SNe Ia may be reduced in favor of AICs. 
The initial set of systems to which the mass loss algorithm was applied was generated by a population synthesis code which has already been used for a number of problems concerning binary star evolution. The detailed description of all the assumptions involved in the synthesis as well as approximations for the dependence of stellar parameters on mass can be found, for example, in Yungelson et al. (1996) and references therein. The occurrence rates of events were obtained by means of Eq. (1) for the binary birthrate from Iben \& Tutukov (1984). The same equation was used for the estimates of HKN and Li \& van den Heuvel (1997).

\section{RESULTS AND DISCUSSION}

\subsection{SN Ia in systems with low-mass giant donors}

As a first step in our modeling, we generated a population of systems which satisfy the criteria for dynamically stable mass exchange. Every system in this sample was then followed throughout its evolution. The values of $\xi_{a d}$ as a function of the relative mass of the core were obtained from Hjellming \& Webbink (1987) and the retention efficiency $\alpha$ was

estimated at each time step as $\dot{M}_{c r} / \dot{M}$. For $\dot{M}_{c r}$ we adopted the data from Iben \& Tutukov (1989).

If initially the donor stars were thermally unstable, then they were stripped of mass until Eq. (2) became valid, according to the prescription given in $§ 2.2$. For stars which were able to evolve on a nuclear timescale initially, or entered this phase after a "thermal" timescale mass loss phase, we used the dependence of the radius, luminosity, and core growth rate on the mass of the core (in solar units and years), derived by Iben \& Tutukov (1984) for solar chemical composition stars:!

$$
R \approx 10^{3.5} M_{H e}^{4}, \quad L \approx 10^{5.6} M_{H e}^{6.5}, \quad \dot{M}_{H e} \approx 10^{-5.36} M_{H e}^{6.6} .
$$

Combining the first and the third expressions in (3) with Eq. (2), we obtained the mass loss rate and the timescale of the evolution (which is controlled by nuclear burning). Formulae (3) give values of $\dot{M}$ which are in very good agreement with those obtained by Webbink, Rappaport \& Savonije (1983; the latter were used by HKN). The integrations were continued up to the point when one of the following situations was encountered.

(i) The hydrogen-rich envelope of the dwarf was exhausted, it became a He white dwarf, but the accretor did not succeed to reach $M_{C h}$ or to accumulate a "critical" mass of He [see option (iii)].

\footnotetext{
${ }^{1}$ Here we correct a misprint in the power of the $L\left(M_{H e}\right)$ dependence in Yungelson et al.
} (1996) 
(ii) The accretor reached $M_{C h}$. Depending on the initial mass of the accretor this event was classified as a SN Ia or an AIC.

(iii) A "critical" mass of He, equal to $\Delta M_{H e}=0.15 M_{\odot}$, was accumulated on top of the accretor due to hydrogen burning, and the accretion rate at that instant was below $3 \times 10^{-8} \mathrm{M}_{\odot} \mathrm{yr}^{-1}$. Such events were classified as ELDs, which may also be responsible for SN Ia (e.g. Livne \& Glasner 1990; Woosley \& Weaver 1994; Livne \& Arnett 1995). If the accumulation of $0.15 M_{\odot}$ of He occurred at a higher $\dot{M}$, we assumed that the ensuing flash was not accompanied by a significant mass loss (Kato, Saio, \& Hachisu 1989). Note, that this assumption is at variance with that made in our earlier papers, where we prescribed some mass loss to accompany such flashes (see e.g. Fig. 2 in Yungelson et al. 1995). The reason for the present assumption was to make our model as similar as possible to that of HKN.

Table 1 summarizes the results of several runs performed for different sets of assumptions. Note that in the Table, we have labeled the events of accumulation of $0.15 M_{\odot}$ as ELD even though the actual outcome is not certain. Models 1 to 5 correspond to the case in which we followed the HKN prescription for the selection of candidate systems: only white dwarf plus red giant systems with a positive denominator in Eq. (2) for $\alpha=0$ were considered for subsequent evolution. Model 1, is in fact a strict reproduction of the HKN model. No "explosive" events (of any kind) were obtained in this model. The reason can be understood by the following estimate. Systems, which have at RLOF $q \lesssim 1.15$ must contain relatively massive white dwarfs. A $\sim 1 M_{\odot}$ white dwarf descends from a main sequence star of 7-8 $M_{\odot}$. This means that in the common envelope stage (in which white dwarf was formed), the separation of the components had to decrease by a factor $a_{f} / a_{0} \sim \alpha_{c e}\left(M_{W D} / M_{1}\right)\left(M_{2} / M_{e n v}\right) \sim 1 / 40-1 / 50$, if $M_{2} \sim 1 M_{\odot}$ and $\alpha_{c e}=1$ [we apply for this estimate Eq. (17) of Iben and Livio (1993) for the variation of separations in common envelopes; here $a_{f}$ and $a_{o}$ are the final and initial separations, respectively, and $\alpha_{c e}$ is the common envelope efficiency parameter]. The widest, but still close in the evolutionary sense, systems with $7 M_{\odot}$ primaries and $1 M_{\odot}$ secondaries have orbital separations of $\sim 1500 R_{\odot}$. After the first common envelope stage this separation is reduced to $a_{f} \sim 38$ $R_{\odot}$. Subsequently, at $\mathrm{RLOF}$, the radius of the donor is $\sim 14.5 R_{\odot}$ and $M_{H e} \approx 0.26 M_{\odot}$. However, Roche-lobe filling stars with such small helium cores lose mass at rates which hardly ever exceed $\sim 10^{-7} \mathrm{M}_{\odot} \mathrm{yr}^{-1}$ (see e.g. Fig. 1 in Kraicheva et al. 1986). Consequently, instead of accumulating matter (by steady hydrogen burning), the accretors enter a regime of thermonuclear runaways and are unable to grow in mass. In the model calculations, the most massive cores of the donors at RLOF did not exceed $\sim 0.3 M_{\odot}$. This means that in its original form, the HKN model (if combined with a value of $\alpha_{c e}=1$, which we consider as "standard", e.g. Rasio \& Livio 1996) is unable to produce any SN Ia.

Our choice of $\alpha_{c e}=1$ as a "standard" value is based on the success of attempts to model, by means of a population synthesis, the numbers and main parameters of such different constituents of the binary star population of the Galaxy as Wolf-Rayet stars, massive X-ray 
binaries, symbiotic stars, cataclysmic variables, double degenerates of different kinds and to obtain reasonable agreement with observations. However, the estimate of $a_{f} / a_{0}$, which is based on rough energy budget considerations may not be very accurate. Furthermore, different formulations of the equation for $a_{f} / a_{0}$ are available in the literature and as a result, even the value of $\alpha_{c e}=1$ may correspond to different amounts of energy deposited into the common envelope in different studies (see Livio 1996b for a discussion). Therefore, it is worthwhile to study the influence of variations in $\alpha_{c e}$ on the rate of SN Ia.

It is evident that a reduction in $\alpha_{c e}$ would result in an even more severe upper limit on the masses of He cores at RLOF. Results of varying $\alpha_{c e}$ between 1 and 20 are given in rows 2 to 5 of Table 1 . Clearly, the conditions for accumulation of $M_{C h}$ become more favorable with the increasing range of the masses of He cores of the donors. Even for $\alpha_{c e}=2$ some SN Ia appear. However, even in the relatively "generous" case of $\alpha_{c e}=10$ the occurrence rate of SN Ia is still two orders of magnitude below the inferred Galactic rate. The upper panel of Fig. 2 shows the position of successful systems in the plane of the initial mass of the accretor $M_{a}$ - initial mass of the donor $M_{d}$, for $\alpha_{C E}=10$, in the HKN model. The figure demonstrates that actually, the original HKN model, if it works at all, is favorable not for SN Ia but for AICs.

Rows 6 to 9 of Table 1 show results of computations for the model of HKN modified to take into account the initial "thermal" stage of mass exchange, as described in $\S 2.2$, for a number of scaling factors for $\dot{M}$. The immediate result is the appearance of SN Ia even for $\alpha_{c e}=1$, for which the original HKN model failed. The results for our version of the model are clarified by Fig. 3, in which we plotted the evolution of the donor and accretor masses for a $1.75+0.85 M_{\odot}$ system, with $a_{0}=10$ and $20 R_{\odot}$, for $\alpha_{t h}=0.5$. In the first case, the mass of the He core of the donor at RLOF is $\sim 0.19 M_{\odot}$. Therefore, under our assumptions, this system is not subject to the restrictions imposed by the requirement of dynamical stability and the donor begins to experience mass loss on a thermal timescale. For $\alpha_{t h}=0.5$ the mass transfer rate is $\sim 6.6 \times 10^{-7} \mathrm{M}_{\odot} \mathrm{yr}^{-1}$. For this value of $\dot{M}$ and with $M_{a}=0.85 M_{\odot}$ the initial retention efficiency is $\alpha \sim 0.6$, i.e. $40 \%$ of the accreted matter is blown off from the system. With increasing $M_{a}$, the retention efficiency also increases and it becomes equal to unity when $M_{a} \approx 1.225 M_{\odot}$. The total mass lost from the system by the optically thick wind is only about $0.1 M_{\odot}$. The mass of the donor grows to $M_{C h}$ very shortly before Eq. (2) becomes valid, still in the "thermal" stage of mass exchange.

If $a_{0}=20 R_{\odot}$, the radius and luminosity of the donor at RLOF are higher and for $\alpha_{t h}=0.5$ the initial mass transfer rate is $\dot{M} \approx 2 \times 10^{-6} \mathrm{M}_{\odot} \mathrm{yr}^{-1}$. The initial retention efficiency is only $\sim 0.2$. The mass of the donor drops faster than in the former case, but the rate of growth of $M_{a}$ is the same, because it is limited by the maximum stable hydrogen burning rate. When the component masses reach the values of $M_{d} \approx 1.05 M_{\odot}$ and $M_{a} \approx 1.0 M_{\odot}$, Eq. (2) becomes valid (formally discontinuously). $\dot{M}$ is initially very slightly above $3 \times 10^{-8} \quad \mathrm{M}_{\odot} \mathrm{yr}^{-1}$. At this $\dot{M}$, the accretor is still able to grow in mass despite nova explosions (according to the calculations of Prialnik \& Kovetz (1995) the results of which 
are incorporated into our code). The retention efficiency is close to 0.05. At the instant when $\dot{M}$ drops below $3 \times 10^{-8} \mathrm{M}_{\odot} \mathrm{yr}^{-1}$, the mass of the accretor is $1.004 M_{\odot}$ and, formally, the criterion for an ELD is satisfied. It is important to note that most of the mass of the "critical" He layer is accumulated at high $\dot{M}$.

The lower panel of Fig. 2 shows the position of systems which accumulate $M_{C h}$ in the initial $M_{a}-M_{d}$ plane, in a modified HKN model, with $\alpha_{t h}=0.5, \alpha_{c e}=1$. It is clear from this Figure that the systems which are able to produce SN Ia are those which experience a thermal timescale mass exchange, i.e. systems, which are slightly evolved off the main sequence. This suggests again that supersoft x-ray sources, in which relatively massive white dwarfs accrete from evolved companions (van den Heuvel et al. 1992; Rappaport, Di Stefano \& Smith 1994; YLTTF; Kahabka 1995; Southwell et al. 1996; Li \& van den Heuvel 1997; Kahabka \& van den Heuvel 1997; Di Stefano 1996; Di Stefano et al. 1997) should be closely examined.

The main difference between the present model and the models considered by Rappaport et al. (1994) and YLTTF is in the fact that we allow for mass transfer rates above the limit for stationary hydrogen burning by assuming that the excess mass leaves the system and stabilizes the mass transfer. This results in wider ranges of initial mass ratios of the components and masses of the cores of the donors. The net effect of this difference is that in about 15 times more systems the white dwarfs can reach $M_{C h}$, compared to previous calculations (compare e.g. models 6 and 10, the latter of which was computed under assumptions similar to those in YLTTF). It is important to note in addition that all the white dwarfs which grew to $M_{C h}$ in the YLTTF model were of the ONe variety, while in the present model $\mathrm{CO}$ white dwarfs also grow to $M_{C h}$.

A comparison between models 10 and 11 shows the effects of varying $\alpha_{c e}$, under assumptions that are similar to those in the YLTTF model.

A comparison of models 6 and 9 shows the effects of varying the scaling factor for the mass loss rate during the "thermal" stage. There exists a value of $\alpha_{t h}$ for which the number of "explosive" events reaches a maximum. If $\alpha_{t h}$ is high, i.e. $\dot{M}$ is high, the retention efficiency is close to zero, but after the mass ratio drops below $\sim 1.15$, the accretion rate also decreases sharply and either a situation which is favorable for an ELD is encountered or an erosion of the already accumulated He layer begins. If $\alpha_{t h}$ is low, the white dwarf may be eroded from the very beginning of the accretion process. A comparison with Fig. 1 of $\mathrm{Li}$ \& van den Heuvel (1997), which shows an example for the behavior of $\dot{M}$ vs. $M_{d}$ from their evolutionary calculations, suggests that $\dot{M}$ may be initially higher than obtained in our simplified models. However, as our trial computations for $\alpha_{t h}=2$ (not included in Table 1) have shown, more mass is then lost in the stage with virtually zero retention efficiency and the rate of explosive events decreases as compared to the $\alpha_{t h}=1$ case.

The criteria for dynamically and thermally stable mass transfer discussed in $§ 2.2$ were derived under the assumption that the stars may be represented by polytropic 
models. While in general this assumption proves to be quite reasonable, it is worthwhile to test its influence on the model. Model 12 gives the results of a trial run with a very "restrictive" model, in which we required that the mass exchange has to be dynamically stable irrespective of the fractional mass of the He core, i.e. the restriction $\xi_{L}<\xi_{a d}$ was imposed on all the candidate systems. This restriction efficiently eliminates all the systems with accretors able to grow to $M_{C h}$.

In contrast, in models 13 to 16 we did not impose any restrictions on the candidate systems. Clearly, these models are the most prolific producers of explosive events. For $\alpha_{t h}=$ 0.2 a SN Ia rate which is consistent with observations is obtained. Varying $\alpha_{t h}$ has the same effects on these models as those discussed above for models 6-9. However, limitations based on the stability of the models have to exist. Trial computations with a full-scale Henyey code (A. Fedorova, private communication) for a $2.0+1.2 M_{\odot}$ system, with $M_{H e 0}=0.4$ $M_{\odot}$ and a zero retention efficiency show that the mass transfer rate increases to $\sim 3 \times 10^{-3}$ $\mathrm{M}_{\odot} \mathrm{yr}^{-1}$ in 80,000 $\mathrm{yr}$ after RLOF. The same behavior is suggested by the computations of Li \& van den Heuvel (1997). This behavior is in complete agreement with the results of Hjellming \& Webbink (1987): $\xi_{a d}$ becomes negative when the fractional mass of the core exceeds $\sim 0.21$. In fact, in our model we rejected such systems because the initial "thermal" $\dot{M}$ was in excess of $10^{-4} \mathrm{M}_{\odot} \mathrm{yr}^{-1}$. The systems with component masses of 2 and $1.2 M_{\odot}$ in Fig. $2 b$ have lower fractional core masses.

Runs 13-16 provide the upper limits for the possible rates of SNe Ia and ELDs from the model under consideration, since the only formal restriction applied was that the mass loss rate by the donor should be $\dot{M} \leq 10^{-4} \mathrm{M}_{\odot} \mathrm{yr}^{-1}$, well over the Eddington critical accretion rate for WDs. These limits are valid irrespective of the mechanism which actually removes the excess of "unaccretable" matter from the system.

\subsection{Comparison to the work of $\mathrm{Li} \&$ van den Heuvel and Di Stefano et al.}

Li \& van den Heuvel (1997) made a series of evolutionary computations using a simplified Eggleton (1971) code, for accretor masses of 1 and $1.2 M_{\odot}$ and a range of donor masses and initial orbital periods of the system. They suggest that the white dwarfs may grow in mass to $M_{C h}$ if $0.9 \lesssim M_{a} / M_{\odot} \lesssim 1.2,2 \lesssim M_{d} / M_{\odot} \lesssim 3.5,0.5 \lesssim P_{\text {orb }} /$ day $\lesssim 6$ or $M_{a} \approx 1 M_{\odot}, 0.9 \lesssim M_{d} / M_{\odot} \lesssim 1.2,70 \lesssim P_{\text {orb }} /$ day $\lesssim 700$. The shorter period range found by Li \& van den Heuvel overlaps with the range found by us, although it is slightly shifted to higher masses of both the accretors and donors (we do not consider here donors with $M_{d} \geq 2.5 M_{\odot}$, since these stars do not have degenerate helium cores and Eq. (2) cannot be applied). Li \& van den Heuvel obtained a rough estimate for the rate of SNe Ia of $2 \times 10^{-3} \mathrm{yr}^{-1}$. However, they did not consider the evolution which leads to the formation of high-mass white dwarfs with low or moderate mass companions. If this is taken into account, the total birthrate of massive white dwarf + main sequence systems, irrespective 
of their fate, in the above range of $M_{a}, M_{d}$, and $P_{\text {orb }}$ and for $\alpha_{c e}=1$ is only $2 \times 10^{-4} \mathrm{yr}^{-1}$. This gives an actual absolute upper limit to the rate of explosive events from the systems considered by Li \& van den Heuvel. Considering their long-period range, systems in that range do not form at all because of the sharp decrease in the orbital separation in the common envelope stage (i.e. for the same reason which makes the original HKN model ineffective).

The upper limit of the orbital period range of successful progenitors of SN Ia found by Li \& van den Heuvel is slightly below the limit found in the present paper (6 to 11 days, depending on the masses of the donor and accretor). This may mean that the stabilizing effect of the large fractional cores of the donors may be weaker than Hjellming \& Webbink (1987) suggest. In such a case, our models actually overestimate the potential occurrence of SN Ia (see the discussion of model 12 above).

Di Stefano et al. (1997) addressed the question of SNe Ia from low-mass binaries by performing Monte-Carlo simulations of the underlying population and evolving it using parameterized results of Henyey-type computations for the estimate of $\xi_{a d}$ as a function of the relative core mass of the donor. The retention efficiency was treated by them as an ajustable parameter and mass loss rates higher than $\dot{M}_{c r}$ were allowed. The mechanism for the removal of excess matter was not specified. The results of Di Stefano et al. are in complete agreement with ours: the rate of SNe Ia is between $9 \times 10^{-5}$ and $1.8 \times 10^{-4} \mathrm{yr}^{-1}$ (for $100 \%$ initial binarity), only a few of percent of the desired rate. The most favored combination of accretor $v s$. donor for the production of SNe Ia is $M_{a} \sim 0.9-1.0 M_{\odot}, M_{d} \sim 2 M_{\odot}$ (compare to Fig. 2). In the most "prolific" case for the production of SNe Ia, when it is assumed that the binaries under consideration may survive the common envelope stage, the supernovae rate is $\sim 0.55 \times 10^{-2} \mathrm{yr}^{-1}$, somewhat higher than in our most prolific case 15 . The reason for this slight difference may be the inclusion of donors with $\dot{M} \gtrsim 10^{-4} \mathrm{M}_{\odot} \mathrm{yr}^{-1}$.

The rate of events involving the accumulation of $0.2 M_{\odot}$ of He on top of the WD in the study of Di Stefano et al. is in all cases higher (by about an order of magnitude) than the rate of events reaching $M_{C h}$, and it may amount to a few tens of percents of the "observed" SN Ia rate (we have higher rates because we consider the accumulation of lower mass of $\mathrm{He})$.

\subsection{Uncertainties}

We would like now to discuss a few important uncertainties. In the discussion above we considered events in which at least $0.15 M_{\odot}$ of He was accumulated on the surface of the accreting white dwarf and $\dot{M}$ became lower than $3 \times 10^{-8} \mathrm{M}_{\odot} \mathrm{yr}^{-1}$, as edge-lit detonations. In fact, this identification represents somewhat of an extrapolation of our current knowledge of the nature of these events. In all the existing calculations of He detonations on top of 
CO dwarfs, either accretion at a constant rate was assumed, or a certain mass of He was exploded by a "piston". These calculations have shown that for the He detonation to cause a C detonation, $\dot{M}$ must be confined to a rather narrow range of $(1-5) \times 10^{-8} \mathrm{M}_{\odot} \mathrm{yr}^{-1}$ (e.g. Woosley \& Weaver 1994). In the case considered in the present study, the rate at which mass is accumulated is that of steady hydrogen burning. Within the uncertainties which exist in the estimates of this rate (for white dwarfs with masses below $\sim 1 M_{\odot}$ ), the critical rate for helium detonation may be considered as being marginally compatible with the lower limit of the stationary hydrogen burning rate $\dot{M}_{s t}$ (although on the lower side). However, the mass is actually accreted at a rate which is much higher than $\dot{M}_{s t}$. This may make the effects of compressional heating important for the thermal history of both the hydrogen and the helium layers. It is thus not entirely clear if all the events identified here as ELDs will indeed result in detonations. It should also be remembered that there does not exist a unique critical mass for the He layer to produce an explosion, rather, it depends (with uncertainties by a factor of a few) on the mass of the white dwarf, its temperature, and $\dot{M}$ (e.g. Kawai, Saio, \& Nomoto 1989; Iben \& Tutukov 1989; Limongi \& Tornambè 1991; Woosley \& Weaver 1994). Another point that should be made is that a formal consideration of Eq. (2) suggests a quite abrupt decrease in $\dot{M}$ from the high "thermal" value to the low "nuclear" one (the denominator changes its sign and it is close to 0). In more realistic models, this transition has to be smooth (see e.g. Li \& van den Heuvel 1997). However, an important conclusion of the present study is, that if the steady accumulation of $\mathrm{He}$ is possible and if it really results in edge-lit detonations which produce SN Ia, then semidetached systems with low mass (sub)giant donors may be the main channel for SN Ia.

In the context of the possible contributions of $M_{C h}$ accumulations and ELDs to the rate of SN Ia it is instructive to consider the age distribution of the two types of events (Fig. 4). The events, termed by us as SN Ia, happen in systems with massive white dwarfs and relatively massive companions, and $M_{C h}$ is accumulated in them shortly after the donor leaves main-sequence. Hence, the typical age of these systems has to be the main-sequence lifetime of $\mathrm{a} \sim 1.8-2.2 M_{\odot}$ star - of the order of $\sim 10^{9} \mathrm{yr}$. Edge-lit detonations on the other hand, as discussed above, occur if the white dwarf accumulates some mass at high $\dot{M}$ (but not reaching $M_{C h}$ ) and then $\dot{M}$ declines to below $3 \times 10^{-8} \mathrm{M}_{\odot} \mathrm{yr}^{-1}$. This requires lower mass donors (typically $\left.\sim 0.6-0.8 M_{\odot}\right)$, lower mass accretors $\left(\sim(1-2) M_{\odot}\right)$ and a long stage of nuclear-burning powered mass exchange. Hence, these systems have typically to be older. In stellar populations with a constant star formation rate the incidence of $M_{C h}$ accumulations "saturates" in $\sim 10^{9} \mathrm{yr}$. Their typically younger age also suggests that these systems are not the progenitors of SNe Ia in elliptical galaxies, which have short initial bursts of star formation. The same is true for the systems producing ELDs, although their typical ages are in the range of 1 to 6 Gyr.

In Table 1 we also give the total number of objects under consideration, $N_{\text {obj }}$, in the Galaxy. All the objects are semidetached systems in which white dwarfs are either burning hydrogen steadily or are experiencing hydrogen shell flashes of the nova type [at the low 
end of the $\dot{M}$ predicted by Eq. (2)]. If the position of the photosphere in the wind is at a radius smaller than $\sim 0.07 R_{\odot}$, the accreting white dwarf may radiate in supersoft X-rays. The upper limit on the number of supersoft X-ray sources represented by the entries in col. (7) of Table 1 is not significantly different from estimates of the number of Galactic supersoft sources, especially if one takes into account absorption effects (e.g. Di Stefano \& Rappaport 1994; YLTTF).

Finally, in col. (8) of Table 1 we list the number of hydrogen flashes on accreting white dwarfs (per year) which may be expected from the systems under consideration. Note, that we included only systems which entered the regime of non-steady burning after passing through a stage of steady accumulation of He. Systems which had $|\dot{M}| \leq\left|\dot{M}_{s t}\right|$ ab ovo were rejected from the sample because they were not interesting as SN Ia progenitors. The number of flashes was estimated, like in Yungelson et al. (1995, 1996), on the basis of models for the accretion of hydrogen onto bare CO white dwarfs (Prialnik \& Kovetz 1995). However, the existence of the He layer does not change the expected rates significantly (compare the critical ignition masses $\Delta m_{a c c}$ from Prialnik \& Kovetz (1995) with $\Delta m_{a c c}$ for white dwarfs with He surface layers from Prialnik \& Livio (1995); the latter are higher only by 30 to $50 \%$ than the former). If we identify these flashes with novae, an important property of these novae is that their ejecta should be enriched in He, but should have solar metal abundances; such novae are known to exist (Livio \& Truran 1994). It should be noted that some of the models in Table 1 produce higher rates of novae than the observed one [even though the latter is highly uncertain (e.g. Shafter 1997)]. Since the calculated rates are definitely lower limits (due to the rejection procedure mentioned above), the reality of the associated models should be regarded as questionable.

The uncertainty in the results due to potential differences in the distribution of binaries over mass ratios or initial separations is not expected to exceed $\sim 20-30 \%$, unless an initial mass-ratio distribution which favors low-mass companions is chosen (see e.g. the study of supersoft X-ray sources by Di Stefano et al. (1994)). As it was shown above, the dependence of the results on the common envelope parameter may be much more important. Since the most prolific producers of SNe Ia are stars with $M \sim 2 M_{\odot}$, the effects of such processes as angular momentum loss via magnetic stellar winds are not important for them.

\subsection{Supernovae in a cosmic perspective}

Recent progress in the understanding of the cosmic history of the star formation rate (SFR; Connolly et al. 1997; Madau et al. 1996; Madau 1997; Pei \& Fall 1995) makes it worthwhile to attempt to gain at least a qualitative insight into the evolution of the supernova rate with redshift $z$. In view of the uncertainties that are still involved, we approximate the Madau et al. plot for the SFR by two straight lines, which gives a peak in the SFR at $z \sim 1.14$. This is almost at the exact position of the peak in the SFR 
as obtained using photometric redshifts (Connolly et al. 1997). We should note that the models suggested so far for the cosmic metal enrichment rate (or star formation rate) still do not fit well all the points in the $\operatorname{SFR}(z)$ diagram (see e.g. Fig. 3 in Connolly et al.)

In our present, highly simplified approach, we have made two crude assumptions. First, we assume that all the galaxies follow the same SFR history. Second, we assume that stellar evolution (which has really been calculated for stars of solar composition) is the same in all the galaxies, irrespective of the epoch in the history.?

Three types of events were considered. First, supernovae descending from massive stars: with a main sequence mass $M \geq 11.4 M_{\odot}$ in close binaries and $M \geq 10 M_{\odot}$ otherwise. These may be identified with SN II, Ib, Ic depending on the presence or absence of a (tenuous) hydrogen envelope at the moment of core collapse. The above assumptions on the range of progenitor masses give for the present $(z=0)$ total rate of SN II, Ib, Ic a value close to $0.02 \mathrm{yr}^{-1}$, which is consistent with observational estimates (e.g. van den Bergh 1991; Cappellaro et al. 1997). The second class included mergers of double degenerates, which we still consider as one of the best progenitor candidate classes for most SN Ia (see Branch et al. 1995 for a discussion). The model produces a rate of mergers with a total mass exceeding $M_{C h}$ in the Galaxy (if a constant SFR for a Hubble time is assumed) of $0.003 \mathrm{yr}^{-1}$, again in agreement with the rates suggested for Sbc-Sc type galaxies (e.g. Cappellaro et al. 1997). The third class included the events of accumulation of $0.15 M_{\odot}$ of He on top of white dwarfs accreting from nondegenerate helium companions. The rate of such events in the Galaxy (under the assumption of a constant SFR) is $\sim 4.5 \times 10^{-3} \mathrm{yr}^{-1}$, i.e. if this channel indeed produces SN Ia, then it can account for the majority of the events. The assumption of a constant SFR may be reasonable, since the systems in the second and third classes are mostly younger than several $10^{9}$ yr (Yungelson \& Tutukov 1997). The values of $h=0.5$ and $q_{o}=0.5$ were assumed for the cosmological parameters.

Fig. 5 gives the history of the above types of SNe as a function of $z$ and look-back time. As could be expected, the curve for SN II, Ib, Ic closely follows the SFR because the age of these systems is $\lesssim 2 \times 10^{7} \mathrm{yr}$. The curve for mergers of double degenerates is somewhat delayed (shifted to a lower $z$ ), because the age of the youngest SN Ia is $\sim 10^{8} \mathrm{yr}$ (i.e. the age of the typical progenitor stars, of 5-10 $M_{\odot}$, plus the delay before gravitational waves radiation brings the components into contact). The systems with nondegenerate helium rich donors in which a potentially explosive accumulation of He occurs are again young. The range of masses of the primaries in the progenitors of the latter systems partially overlaps with that for the double degenerates, but the components are typically closer after the white dwarf plus nondegenerate star form.

While at $z$ close to 1 the helium ignitors dominate (in terms of the rate), at $z$ close to 0

\footnotetext{
${ }^{2}$ A similar approach was used e.g. by Canal, Ruiz-Lapuente, \& Burkert (1996) and by Yungelson \& Tutukov (1997).
} 
the central carbon ignitors (double degenerates) begin to dominate, because of the existence of a reservoir of pairs formed at earlier epochs. For the systems with nondegenerate helium donors which are candidates for ELD such a reservoir does not exist, because it is limited by the lifetime of low-mass He-stars, $T_{H e} \lesssim 10^{9} \mathrm{yr}$.

The actual slope of the curves for the incidence of supernovae at $z \sim 1-2$ may be slightly less steep than shown in Fig. 5, since our simple approximation for the SFR in this interval may be steeper than the actual one.

We also show in Fig. 5 the ratio of the rate of mergers (SN Ia) to the rate of explosions of massive stars (SN II, Ib, Ic). As can be seen from the figure, this ratio is nearly constant for $z \gtrsim 1.2$, but it increases quite significantly between $z=1$ and the present. The reason for this increase is the build-up of a reservoir of degenerate pairs in the earlier epochs (higher $z$ ).

If $\mathrm{SNe}$ Ia were central ignitors that descend from low-mass semidetached systems of the type analyzed in the present paper, then their formation history would follow the SFR history (as a function of $z$ ), because the age of these systems is typically below $1 \mathrm{Gyr}$ and no reservoir exists for them (Fig. 4; see also Yungelson \& Tutukov 1997). If on the other hand, ELDs in low-mass systems really produce supernovae, then their dependence on redshift or look-back time may be more like that of central carbon ignitors (due to the merger of double degenerates), because of the existence of a considerable fraction of the systems in which ELDs may occur several Gyr after formation (Fig. 4).

\section{CONCLUSIONS}

On the basis of the calculations presented in this paper we can draw the following (somewhat tentative, in view of the uncertainties) conclusions:

1. The incorporation of the possibility of mass loss by the accreting white dwarfs, via optically thick winds, allows to increase the number of systems which are able to burn hydrogen steadily. However, if only the nuclear burning-controlled phase of the evolution of the donors is considered [with $q \lesssim 1.15$; governed by Eq. (2)], as suggested by Hachisu, Kato, \& Nomoto (1996), the rate at which white dwarfs grow in mass to $M_{C h}$ does not exceed a few percent of the currently estimated occurrence rate of Galactic SN Ia (see rows 1 to 5 of Table 1$)$.

2. The inclusion of a phase of mass exchange on a thermal timescale of the donor, which precedes the "nuclear" phase while $q>1.15$, results in an occurrence rate of SN Ia of the order of $\sim 10^{-4}$ (see rows 6 to 9 of Table 1). If the Galactic SN Ia rate is indeed $\sim 3 \times 10^{-3} \mathrm{yr}^{-1}$, then it appears that this channel is not the main route to SN Ia, but it may account for about $10 \%$ of the events (significant uncertainties still exist however). This may be compatible with the growing suspicion that there could be several channels of evolution 
leading to SN Ia and the possibility that there exists a diversity in the parameters of the observed supernovae (see e.g. Branch et al. 1995 for a discussion). As potential evidence for the latter suggestion one may consider the differences in the luminosities of SNe Ia in volume limited samples (Schaefer 1996).

3. An interesting property of the model which involves an optically thick wind phase is the potential presence of non-negligible amounts of circumbinary hydrogen. Deep searches for early radio emission would thus be able to test for the presence of such hydrogen in future SNe Ia (Boffi \& Branch 1995).

4. One may consider two additional situations in which the accretion of hydrogen at relatively high $\dot{M}$ can occur onto white dwarfs, namely, in cataclysmic variables and in symbiotic stars. In the former systems this may happen just after RLOF by the donor, while in the latter, when their donors (losing mass by a wind) are very close to the end of the symbiotic star stage (see e.g. Yungelson et al. 1995, 1996). However, it has been shown that the number of events in cataclysmic variables is at the level of $10^{-5} \mathrm{yr}^{-1}$ and it is almost insensitive to variations in the criteria for dynamically stable mass exchange and steady hydrogen burning. The reason for this insensitivity is the fact that for low mass stars, $\dot{M}_{t h}$ is of the order of $10^{-7} \mathrm{M}_{\odot} \mathrm{yr}^{-1}$, and hence, not many systems are rejected from the sample because of accretion rates exceeding the maximum allowing for steady hydrogen burning.

Concerning symbiotic stars, Yungelson et al. (1995, 1996) did not consider the stage of RLOF, because in an overwhelming majority of the systems, at the beginning of RLOF the mass ratios of the components do not satisfy the criteria for dynamically stable mass exchange. However, when these criteria are satisfied, the mass exchange rate may be as high as $\dot{M} \sim 10^{-6}-10^{-5} \mathrm{M}_{\odot} \mathrm{yr}^{-1}$ (see e.g. de Kool et al. 1986; Pastetter \& Ritter 1989). Consequently, we updated the code to take into account the possibility of stable mass exchange and mass loss from the white dwarfs via optically thick winds (as described in the present paper). We found, however, that the rate of accumulation of $M_{C h}$ still remained at the level of $\sim 10^{-6} \mathrm{yr}^{-1}$ and the rate of accumulation of $0.15 M_{\odot}$ of He at the level of $\sim 10^{-4} \mathrm{yr}^{-1}$, like in the above quoted studies. The reasons for these low rates are: the paucity of systems which satisfy the criteria for dynamically stable mass exchange, the short duration of this stage (if it happens at all), and the virtual absence of white dwarfs with masses in excess of $1 M_{\odot}$ in the zero-age population of symbiotic systems.

The possible presence of optically thick winds from the white dwarfs in symbiotic stars does not influence the number of systems which go through two common envelope stages and form double degenerates with $M_{t o t} \geq M_{C h}$ (which later merge due to gravitational wave radiation). The reason is that the progenitors of these systems are relatively massive: 4-10 $M_{\odot}$. As trial runs of the code show, at the second RLOF, despite the stellar wind mass loss, the companions to the first-born white dwarfs are still sufficiently massive to 
experience a dynamically unstable mass loss.[?

5. The rate of SN Ia as a function of redshift generally follows the star formation rate curve, with a small shift of the peak (by about $\Delta z \sim 0.1$ ) towards lower redshifts. The ratio of the rates of SN Ia to SN II, Ib, Ic stays flat for $z \gtrsim 1$, but increases from $z \sim 1$ towards lower redshifts, and it is higher at $z \sim 0$, by about a factor 4 , than its value at $z \sim 1$. A similar trend is expected for the nucleosynthetic abundances ratio of, for example, $[\mathrm{Fe} / \mathrm{O}]$, but great care should be taken in the interpretation of actual measurements (see e.g. Kulkarni, Fall, \& Truran 1997).

We a grateful to A. Fedorova for the computation of several representative stellar models upon our request. Helpful discussions with A. Tutukov, P. Madau, and P. Kahabka and a critical reading of the manuscript by E. P. J. van den Heuvel and the referee, P. Ruiz-Lapuente, are acknowledged. LY acknowledges the support and hospitality of the ST ScI and support from RFBR grant 96-02-16351. ML acknowledges support from NASA Grant NAGW-2678.

\footnotetext{
${ }^{3}$ We should mention here, in a broader context, the importance of an accurate consideration of the stellar wind mass loss before RLOF for the predicted period distribution of the population of double degenerates.
} 


\section{REFERENCES}

Benz, W., Bowers, R. L., Cameron, A. G. W., \& Press, W. H. 1990, ApJ, 348, 647

Boffi, F. R., Branch, D. 1995, PASP, 107, 347

Branch, D., Livio, M., Yungelson, L. R., Boffi, F. R., \& Baron, E., 1995, PASP, 107, 717

Canal, R., \& Schatzman, E. 1976, A\&A, 46, 229

Canal, R., \& Gutierrez, J. 1997, in White Dwarfs, ed. J. Isern, M. Hernanz, \& E. Garcia-Berro (Dordrecht: Kluwer), p. 49

Canal, R., Ruiz-Lapuente, P., \& Burkert, A. 1996, ApJ, 456, L101

Cappellaro, E., Turrato, M., Tsvetkov, D. Yu., Bartunov, O. S., Pollas, C., Evans, R., \& Hamuy, M. 1997, A\&A, 322, 431

Connolly, A. J., Szalay, A. S., Dickinson, M., SubbaRao, M. U., \& Brunner, R. J. 1997, ApJ, 486, L11

Di Stefano, R. 1996, in Supersoft X-Ray Sources, ed. J. Greiner (Berlin: Springer), p. 193

Di Stefano, R., \& Rappaport, S. 1994, ApJ, 437, 733.

Di Stefano, R., Nelson, L. A., Lee, W., Wood, T. H., \& Rappaport, S. 1997, in Thermonuclear Supernovae, ed. P. Ruiz-Lapuente, R. Canal, \& J. Isern (Dordrecht: Kluwer), p. 147

Dominguez, I., Tornambè, A., \& Isern, J. 1993, ApJ, 419, 268

Eggleton, P. P. 1971, MNRAS, 151, 351

Hachisu, I, Kato, M., \& Nomoto, K. 1996, ApJ, 470, L97 (HKN)

Hjellming, M., \& Webbink, R. 1987, ApJ, 318, 794

Iben, I. Jr., \& Livio, M. 1993, PASP, 105, 1373

Iben, I. Jr., \& Tutukov, A. V. 1984, ApJSS, 54, 355

Iben, I. Jr., \& Tutukov, A. V. 1989, ApJ, 342, 430

Iben, I. Jr., Tutukov, A. V., \& Yungelson, L. R. 1997, ApJ, 475, 291.

Kahabka, P. 1995, A\&A, 304, 227

Kahabka, P., \& van den Heuvel E. P. J. 1997, ARA\&A, 35, in press

Kato, M., \& Hachisu, I. 1994, ApJ, 437, 802

Kato, M., Saio, H, \& Hachisu, I. 1989, ApJ, 340, 509

Kawai, Y., Saio, H., \& Nomoto, K. 1987, ApJ, 315, 229

de Kool, M., van den Heuvel, E. P. J., \& Rappaport, S. A. 1986, A\&A, 164, 73

Kraicheva, Z. T., Tutukov, A. V., \& Yungelson, L. R. 1986, Astrophysics, 26, 167 
Kulkarni, V. P., Fall, S. M., \& Truran, J. W. 1997, ApJ, 484, L7

Li, X.-D., \& van den Heuvel, E. P. J. 1997, A\&A, 322, L9

Limongi, M., \& Tornambé, A. 1991, ApJ, 371, 317

Livio, M. 1996a, in Supersoft Sources, ed. J. Greiner (Berlin: Springer), p. 184

Livio, M. 1996b, in Evolutionary Processes in Binary Stars, ed. R. A. M. J. Wijers, M. B.

Davies, \& C. A. Tout (Dordrecht: Kluwer), p. 141

Livio, M., \& Truran, J. W. 1994, ApJ, 425, 797

Livne, E., \& Glasner, A. S. 1990, ApJ, 361, 244

Livne, E., \& Arnett, D. 1995, ApJ, 452, 621

Madau, P. 1997, in The Hubble Deep Field, ed. M. Livio, M. Fall, \& P. Madau (Cambridge: CUP), in press

Madau, P., Ferguson, H. C., Dickinson, M. E., Giavalisco, M., Steidel, C. C. \& Fruchter, A. 1996, MNRAS, 283, 1388

Mochkovich, R., \& Livio, M. 1990, A\&A, 236, 378

Mochkovich, R., Guerrero, J., \& Segretain, L. 1997, in Thermonuclear Supernovae, eds. P. Ruiz-Lapuente, R. Canal, \& J. Isern (Dordrecht: Kluwer), p. 187

Nomoto, K., \& Kondo, Y. 1991, ApJ, 367, L19

Paczyński, B. 1970, Acta Astron., 20, 47

Pastetter, L., \& Ritter, H. 1989, A\&A, 214, 186

Pei, Y. C. \& Fall, S. M. 1995, ApJ, 454, 69

Prialnik, D., \& Livio, M. 1995, PASP, 107, 1201

Prialnik, D., \& Kovetz, A. 1995, ApJ, 445, 789

Rappaport, S., Di Stefano R., \& Smith, M. 1994, ApJ, 426, 692

Rasio, F. A., \& Livio, M. 1996, ApJ, 471, 366

Refsdal, S., \& Weigert, A. 1970, A\&A, 6, 426

Refsdal, S., \& Weigert, A. 1971, A\&A, 13, 367

Ritossa, C., Garcia-Berro, E., \& Iben, I. Jr. 1996, ApJ, 460, 489

Ritter, H. 1996, in Evolutionary Processes in Binary Stars, ed. R. A. M. J. Wijers, M. B. Davies, \& C. A. Tout (Dordrecht: Kluwer), p. 307

Ruiz-Lapuente, P., Canal, R., \& Burkert, A. 1997, in Thermonuclear Supernovae, eds. P. Ruiz-Lapuente, R. Canal, \& J. Isern (Dordrecht: Kluwer), p. 205

Saffer, R. A. \& Livio, M. 1997, ApJL, submmitted

Segretain, L., Chabrier, G., \& Mochkovich, R. 1997, ApJ, 481, 355 
Shaefer, B. E. 1996, ApJ, 464, 403

Shafter, A. W. 1997, ApJ, 487, 226

Soberman, G. E., Phinney, E. S., \& E. P. J. van den Heuvel 1997, A\&A, in press

Southwell, K. A., Livio, M., Charles, P. A., O'Donoghue, D., \& Sutherland, W. J. 1996, ApJ, 470, 1065

Uus, U. 1970, Nauchn. Inf., 17, 32

van den Bergh, S. 1991, in Supernovae, ed. S. E. Woosley (Berlin: Springer), p. 711

van den Heuvel, E. P. J., Bhattacharya, D., Nomoto, K., \& Rappaport, S. A. 1992, A\&A, 262,97

Webbink, R. F. 1984, ApJ, 227, 355.

Webbink, R. F., Rappaport, S., \& Savonije, G. J. 1983, ApJ, 270, 678

Wheeler, C. J., 1996, in Evolutionary Processes in Binary Stars, eds. R. A. M. J. Wijers, M. B. Davies, \& C. A. Tout (Dordrecht: Kluwer), p. 307

Whelan, J., \& Iben, I. Jr. 1973, ApJ, 186, 1007

Woosley, S. E., \& Weaver, T. 1994, ApJ, 423, 371

Yungelson, L. 1973, Nauchn. Inf., 26, 71

Yungelson, L., Livio, M., Tutukov, A., \& Kenyon, S. J. 1995, ApJ, 447, 656

Yungelson, L., Livio, M., Truran, J. W., Tutukov, A., Fedorova, A. V. 1996, ApJ, 466, 890 (YLTTF)

Yungelson, L., \& Tutukov, A., 1997, in Advances in Stellar Evolution, ed. R. T. Rood \& A. Renzini (Cambridge: CUP), p. 237 
Figure 1. The dependence of the derivatives of the Roche lobe radius $\xi_{L}$ on the mass ratio of the components, for retention efficiencies $\alpha=1$ and 0 (solid curves). The dashed lines give the values of the adiabatic response of stellar radii $\xi_{a d}$, for core mass fractions in condensed polytropes (Hjellming \& Webbink 1987) $m_{c}=0.1962$ and 0.3833 .

Figure 2. The initial masses of accretors and donors in systems which produce SN Ia. Plotted is the value of $\frac{\partial^{2} \nu}{\partial M_{a} \partial M_{d}}$, where $\nu$ is the birthrate of the parent MS system. Upper panel - the HKN model for $\alpha_{c e}=10$, lower panel - the HKN model as modified in the present paper (see text) with a scaling factor for the mass loss rate of $\alpha_{t h}=0.5$. The maximum of the gray scale is the same for both panels and corresponds to $\frac{\partial^{2} \nu}{\partial M_{a} \partial M_{d}}=1.6 \times 10^{-3}$.

Figure 3. The dependence of the donor and accretor masses on time in systems with $a_{0}=10 R_{\odot}$ (upper curve) and $20 R_{\odot}$ (lower curve) in the model with $\alpha_{t h}=0.5$.

Figure 4. Upper panel-The contribution of stars of different ages to the present rate of accumulations of $M_{C h}$ (solid histogram) and of edge-lit detonations (dashed histogram). Lower panel-The time evolution of these events under the assumption of constant star formation rate.

Figure 5. The dependence of the cosmic SN rate on $z$ (panel $a$ ) and on look-back time (panel b). Long-dashed curve - sum of SN II, Ib, and Ic descending from massive stars; short-dashed line - accumulations of $0.15 M_{\odot}$ of He on top of a white dwarf at an accretion rate of $\dot{M} \lesssim 3 \times 10^{-8} \quad \mathrm{M}_{\odot} \mathrm{yr}^{-1}$ in systems with nondegenerate helium donors; solid line - mergers of double degenerates with a total mass greater than $M_{C h}$; dotted line in panel $a$ gives the logarithm of the ratio of the rate of mergers of double degenerates to the rate of explosions of massive stars (a factor -4.5 was added for scaling). 
TABLE 1. Occurrence Rates of SN Ia and Related Events Under Different Model Assumptions

\begin{tabular}{rrllllrr}
\hline \hline $\mathrm{N}$ & $\alpha_{c e}$ & $\alpha_{t h}$ & $\begin{array}{l}\text { SN Ia } \\
\left(\mathrm{yr}^{-1}\right)\end{array}$ & $\begin{array}{l}\text { AIC } \\
\left(\mathrm{yr}^{-1}\right)\end{array}$ & $\begin{array}{l}\text { ELD } \\
\left(\mathrm{yr}^{-1}\right)\end{array}$ & $\begin{array}{r}N_{o b j} \\
(6)\end{array}$ & $\begin{array}{r}R_{N} \\
\left(7 \mathrm{yr}^{-1}\right) \\
(8)\end{array}$ \\
\hline 1 & $(2)$ & $(3)$ & $(4)$ & $(5)$ & 0.0 & 30 & 5 \\
2 & 2 & - & 0.0 & $8 \times 10^{-7}$ & $3 \times 10^{-6}$ & 50 & 9 \\
3 & 5 & - & $3 \times 10^{-7}$ & $5 \times 10^{-6}$ & $5 \times 10^{-6}$ & 80 & 21 \\
4 & 10 & - & $2 \times 10^{-6}$ & $2 \times 10^{-5}$ & $6 \times 10^{-7}$ & 110 & 27 \\
5 & 20 & - & $5 \times 10^{-6}$ & $4 \times 10^{-5}$ & 0.0 & 130 & 32 \\
\hline 6 & 1 & 1 & $7 \times 10^{-5}$ & $5 \times 10^{-5}$ & $3 \times 10^{-3}$ & 6100 & 31 \\
7 & 1 & 0.5 & $2 \times 10^{-4}$ & $4 \times 10^{-5}$ & $3 \times 10^{-3}$ & 8500 & 50 \\
8 & 1 & 0.2 & $1 \times 10^{-4}$ & $2 \times 10^{-5}$ & $4 \times 10^{-3}$ & 9700 & 110 \\
9 & 1 & 0.1 & $1 \times 10^{-5}$ & $7 \times 10^{-5}$ & $4 \times 10^{-3}$ & 9400 & 84 \\
\hline 10 & 1 & - & 0.0 & $8 \times 10^{-6}$ & $1 \times 10^{-5}$ & 400 & 12 \\
11 & 10 & - & 0.0 & $2 \times 10^{-5}$ & 0.0 & 220 & 25 \\
\hline 12 & 1 & 1 & 0.0 & 0.0 & 0.0 & 37 & 3 \\
\hline 13 & 1 & 1 & $9 \times 10^{-5}$ & $7 \times 10^{-5}$ & $5 \times 10^{-3}$ & 11000 & 74 \\
14 & 1 & 0.5 & $3 \times 10^{-4}$ & $8 \times 10^{-5}$ & $6 \times 10^{-3}$ & 16000 & 110 \\
15 & 1 & 0.2 & $1 \times 10^{-3}$ & $5 \times 10^{-5}$ & $7 \times 10^{-3}$ & 20000 & 74 \\
16 & 1 & 0.1 & $8 \times 10^{-4}$ & $2 \times 10^{-5}$ & $9 \times 10^{-3}$ & 25000 & 380 \\
\hline
\end{tabular}

Notes: $\mathrm{N}$-number of model; $\alpha_{c e}$ - common envelope parameter; $\alpha_{t h}$-scaling factor for mass transfer rate; SN Ia-occurrence rate of SN Ia via the accumulation of $M_{C h}$; AIC-occurrence rate of accretion induced collapses; ELD - occurrence rate of edge-lit detonations after the accumulation of $0.15 M_{\odot}$ of He (see text); $N_{o b j}$ - Galactic number of objects; $R_{N}$ - occurrence rate of hydrogen flashes in the nuclear burning controlled evolutionary stage. 


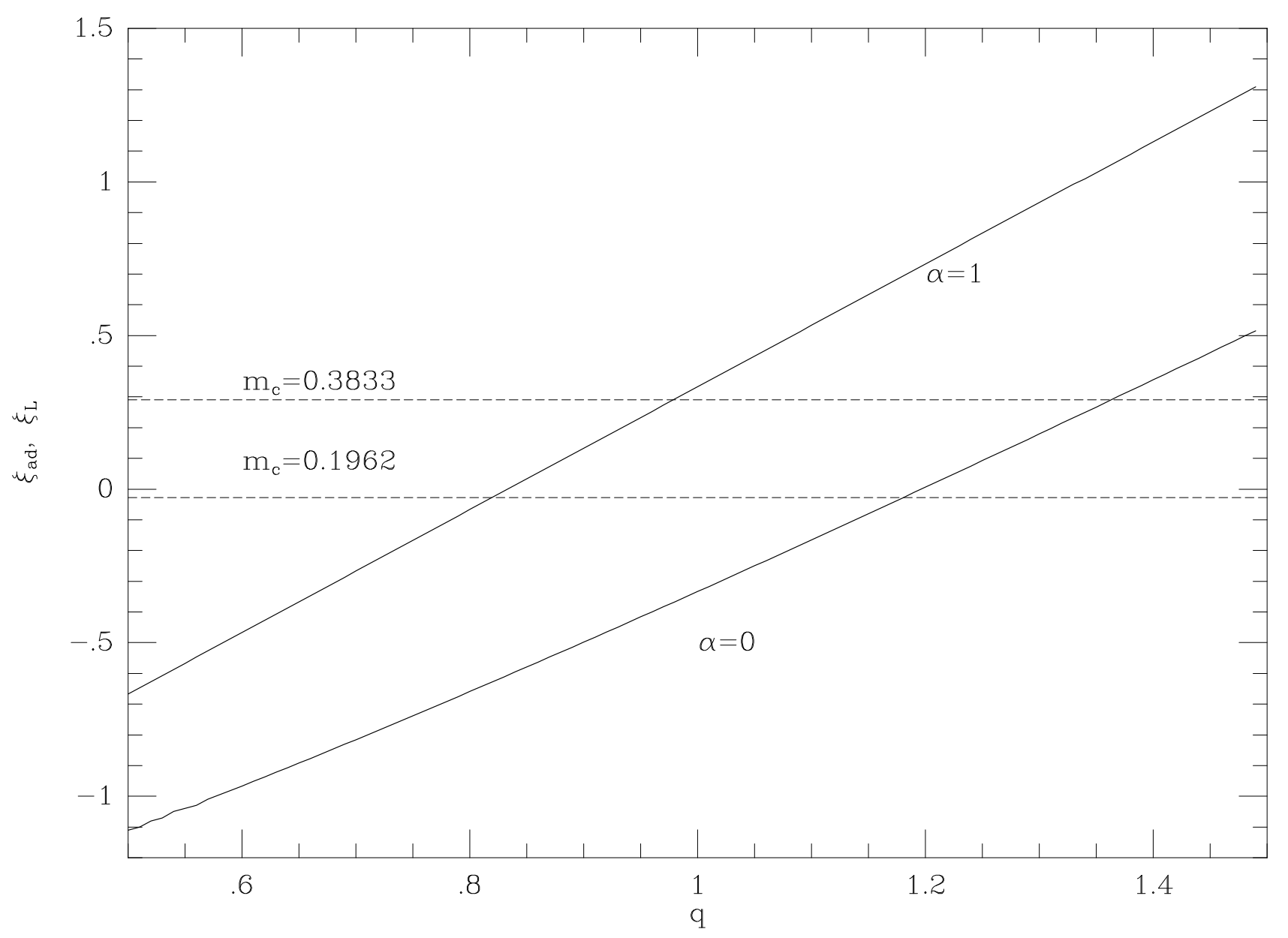

Fig. 1.- 

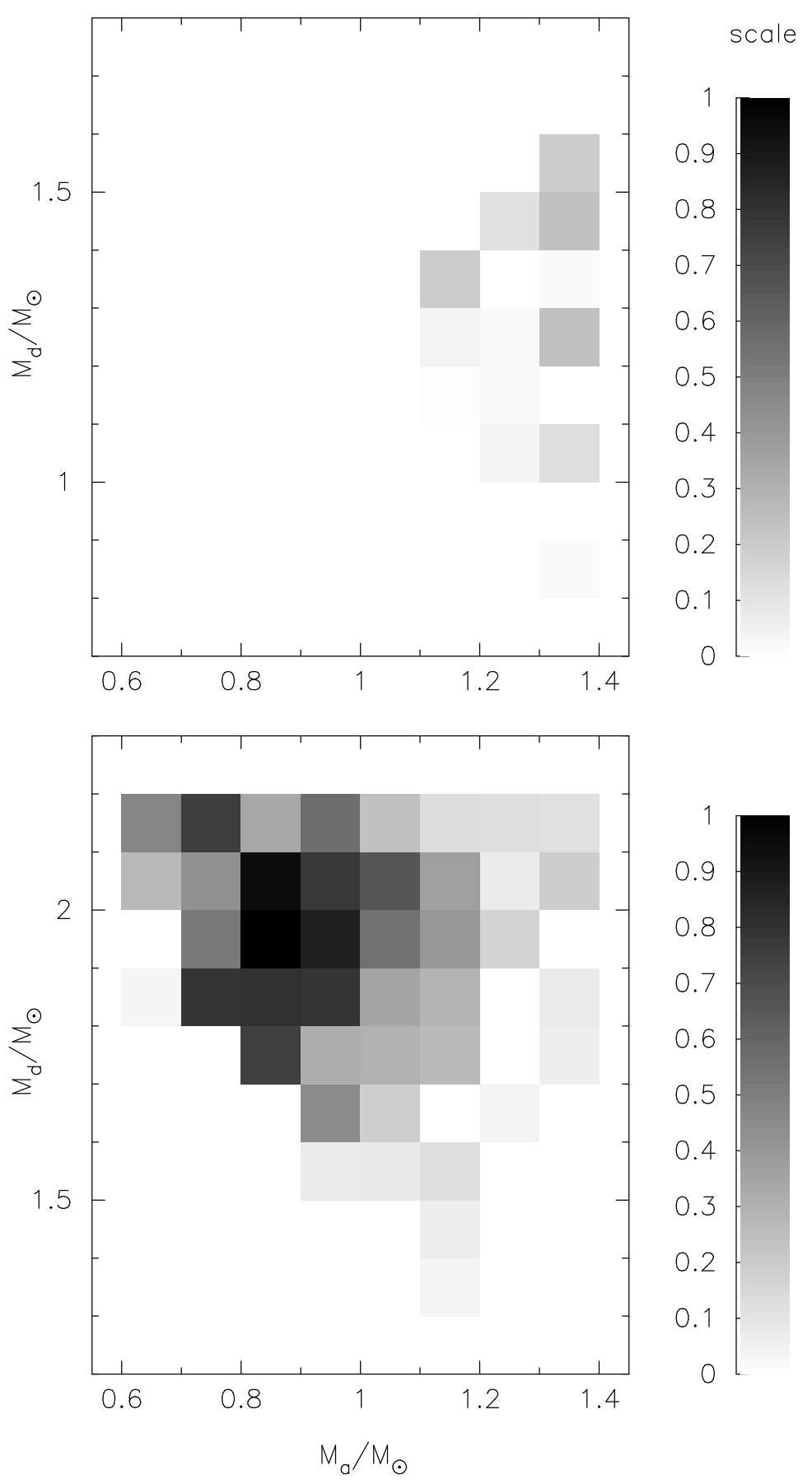

Fig. 2.- 


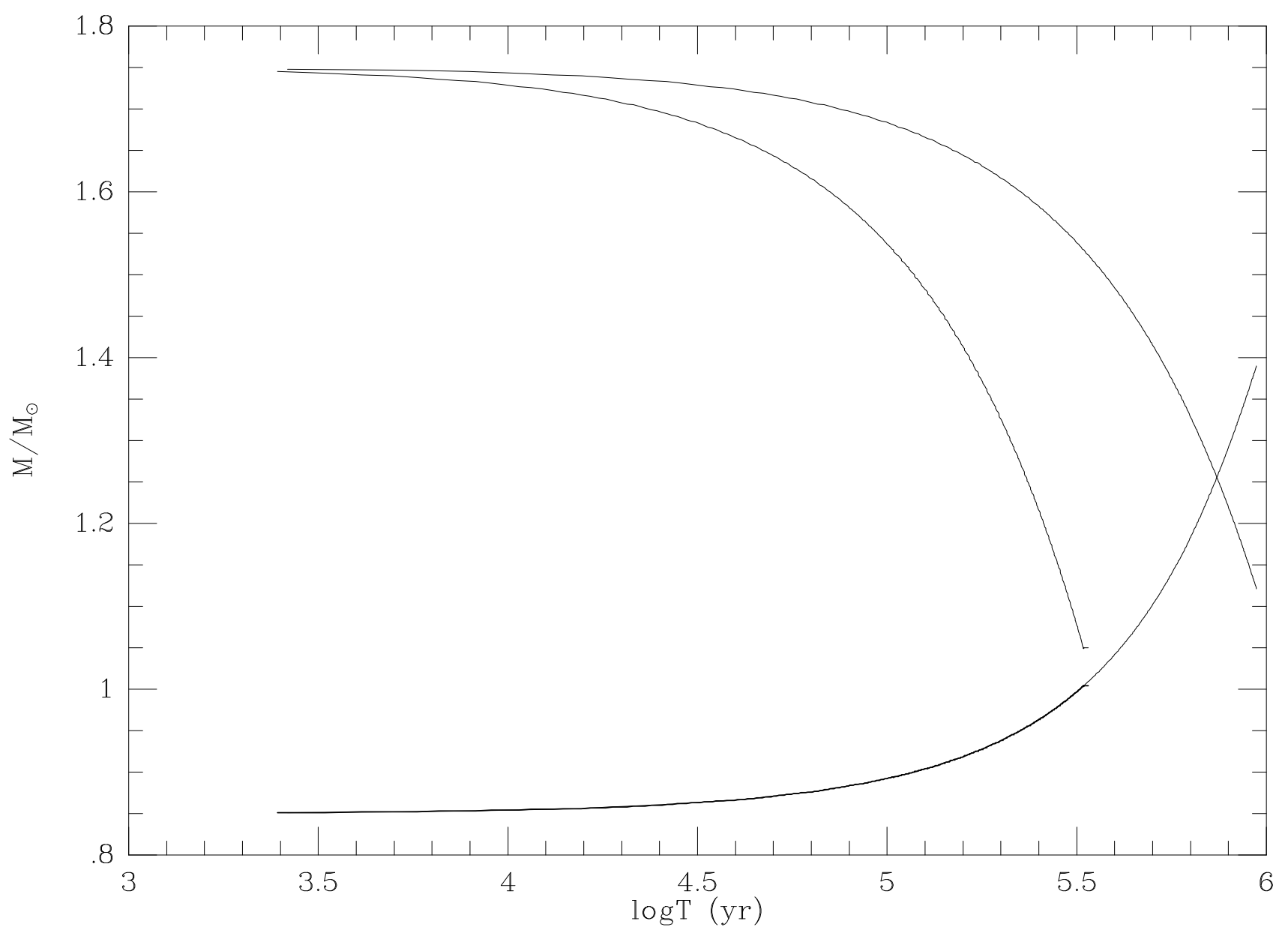

Fig. 3.- 

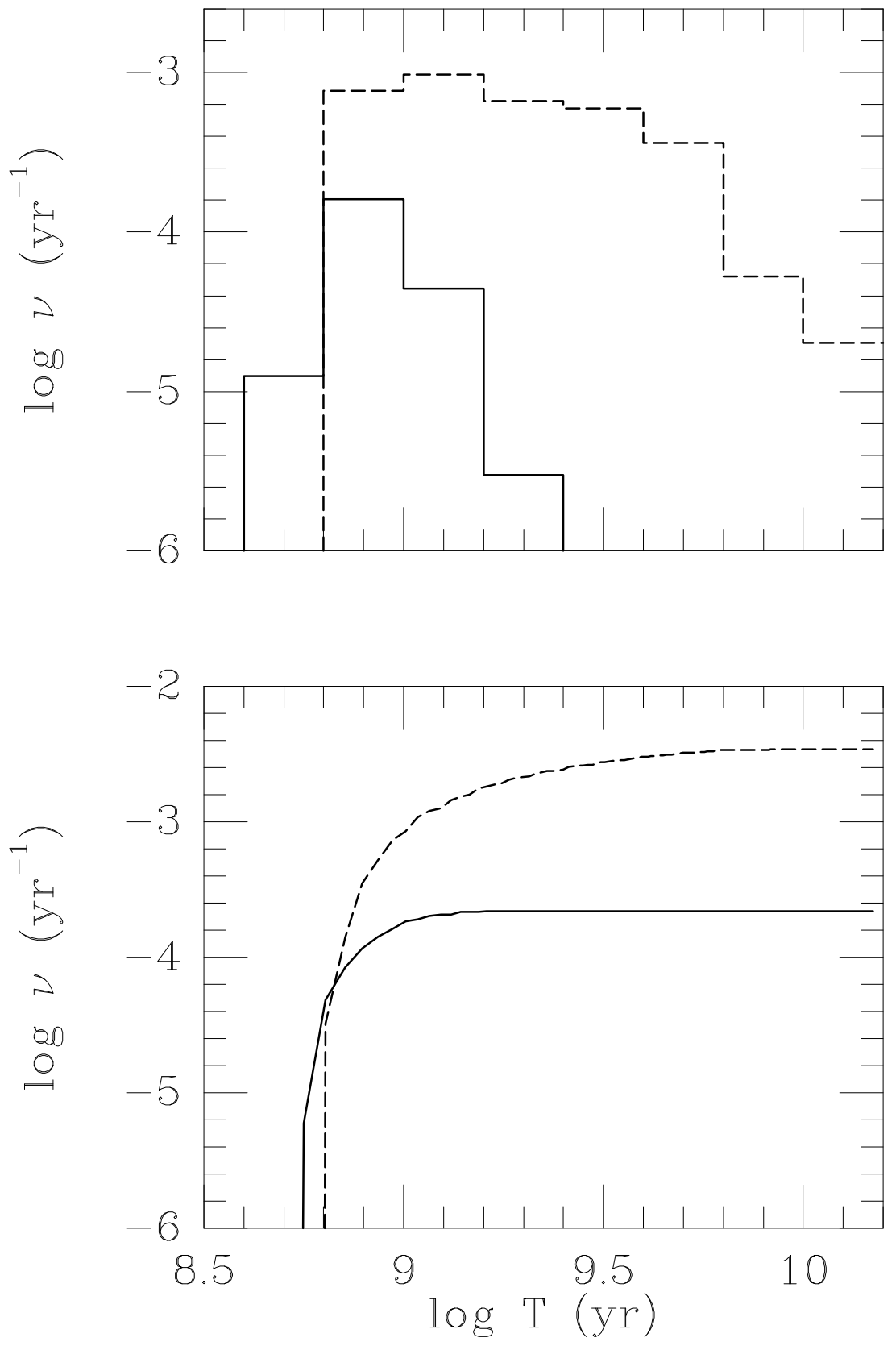

Fig. 4.- 


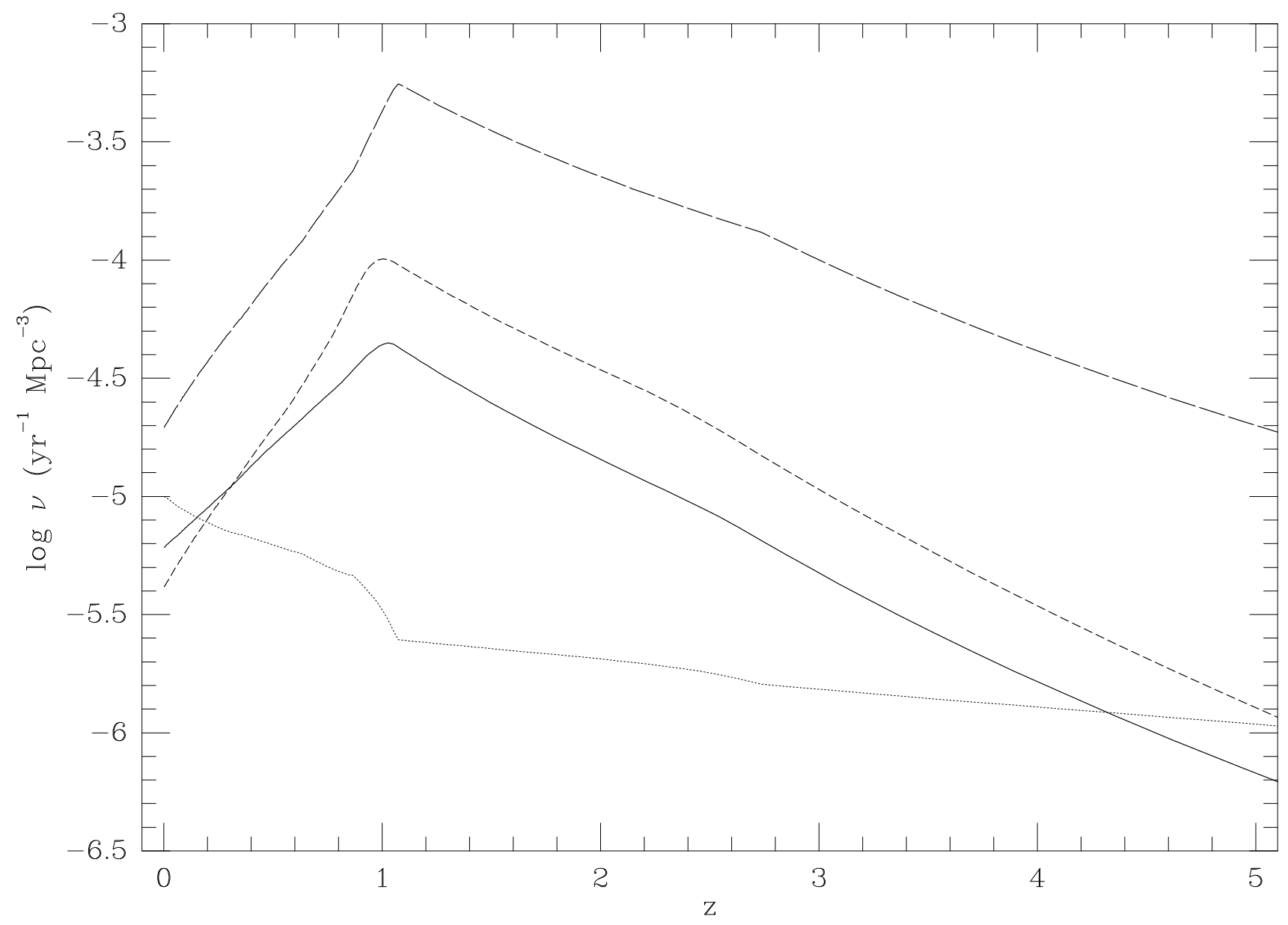

Figure 5a: 


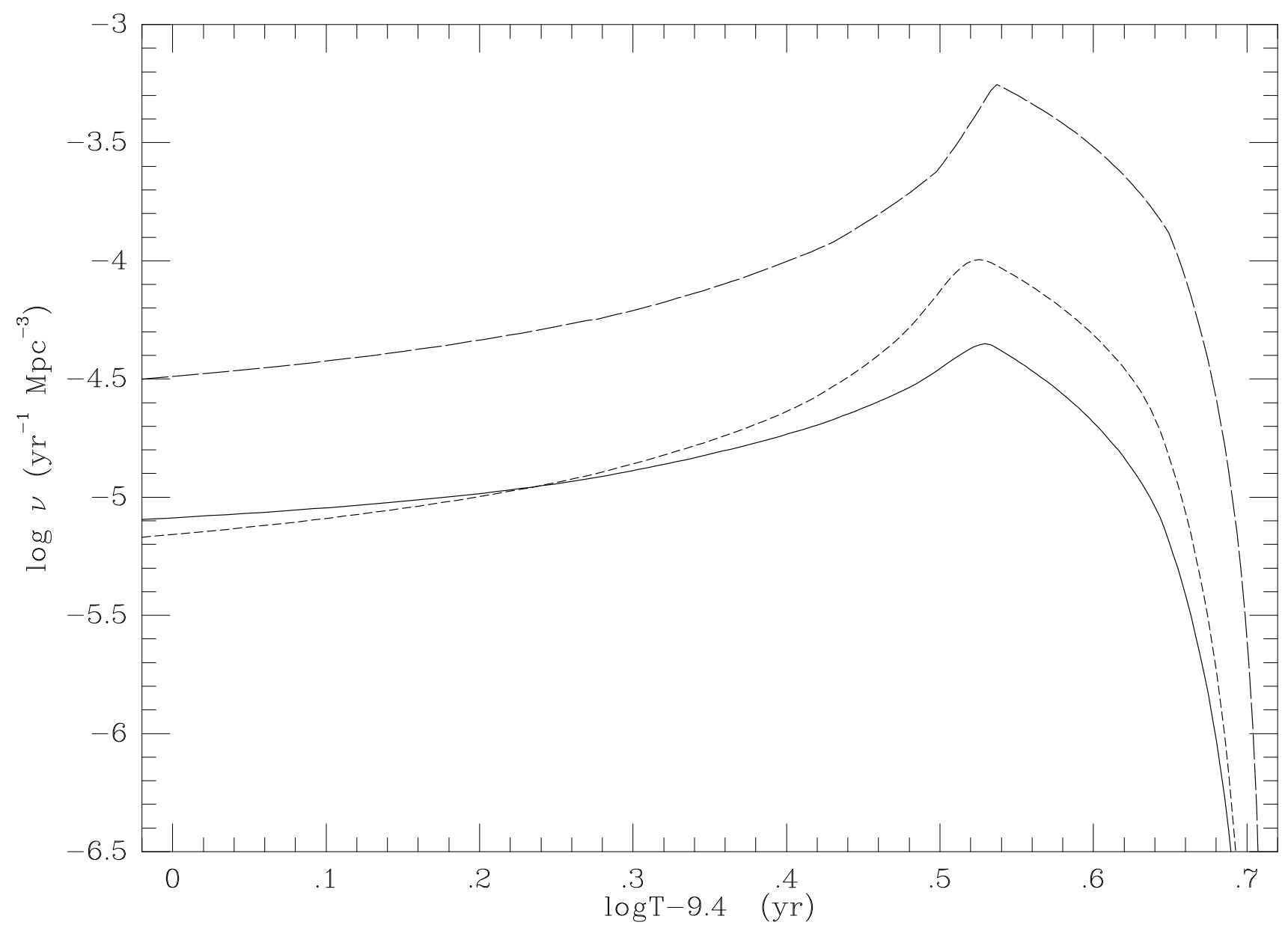

Figure 5b: 